\title{
Facteurs de risque associés à la tuberculose pharmacorésistante dans un centre de référence situé à Toronto (Ontario) au Canada : 2010 à 2016
}

Takashi Hirama ${ }^{1,2,3,4 *}$, Natasha Sabur ${ }^{2}$, Peter Derkach², Jane McNamee ${ }^{2}$, Howard Song ${ }^{2}$, Theodore Marras ${ }^{1,3}$, Sarah Brode ${ }^{1,2,3}$

\section{Résumé}

Contexte : La tuberculose (TB) pharmacorésistante représente une menace majeure pour la santé publique dans le monde. Cependant, aucune étude ne s'est intéressée aux facteurs de risque associés à la pharmacorésistance en Ontario, une province où les immigrants présentent un profil caractéristique. Nous avons évalué les facteurs de risque démographiques et cliniques associés à la TB pharmacorésistante chez les patients traités au Centre de santé West Park situé à Toronto, en Ontario (Canada).

Méthodes : Tous les patients chez qui l'on a diagnostiqué la TB et qui ont été traités au Centre de santé West Park entre janvier 2010 et décembre 2016 ont été pris en compte dans cette étude de cohorte rétrospective. Les caractéristiques des patients atteints d'une TB monorésistante à l'isoniazide (R-INH) et d'une TB multirésistante (MR) ont été comparées à celles des patients atteints d'une TB pharmacosensible à l'aide d'une régression logistique bivariée et multivariée.

Résultats : Les facteurs de risque associés à la TB R-INH étaient l'appartenance à un groupe d'âge plus jeune (moins de 35 ans), l'existence d'antécédents de traitement de la TB, l'absence de diabète et la naissance dans un pays situé ailleurs qu'en Asie du Sud-Est, mais seuls les deux derniers facteurs étaient significatifs dans l'analyse multivariée. En revanche, nous avons constaté que l'appartenance à une génération plus jeune (moins de 65 ans), la naissance dans la région européenne, l'arrivée récente au Canada (moins de 120 mois), l'existence d'antécédents de traitement et l'infection par le virus de l'immunodéficience humaine $(\mathrm{VIH})$ étaient associés à la TB-MR et que, parmi ces facteurs, l'appartenance à un groupe d'âge plus jeune (moins de 35 ans), l'immigration plus récente (moins de 24 mois), l'existence d'antécédents de traitement et l'infection par le VIH étaient significatifs dans l'analyse multivariée.

Conclusion : Ces résultats pourraient être utiles aux cliniciens de la province spécialisés dans la tuberculose, car ils contribuent à éclairer l'antibiothérapie empirique qu'ils prescrivent dans l'attente des résultats de l'épreuve de sensibilité phénotypique aux médicaments, et à faciliter la prise d'une décision quant à la nécessité de demander ou non une épreuve moléculaire rapide de sensibilité aux médicaments.
Cette oeuvre est mise à la disposition selon les termes de la licence internationale Creative Commons Attribution 4.0

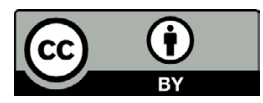

Affiliations

${ }^{1}$ Division de pneumologie, Département de médecine, Université de Toronto, Toronto, ON

2 Département de pneumologie, Centre de santé West Park, Toronto, ON

${ }^{3}$ Division de pneumologie, Département de médecine, Hôpital Toronto Western, Toronto, ON

${ }^{4}$ Département de chirurgie thoracique, Institut du développement, du vieillissement et du cancer, Université Tohoku, Sendai, Miyagi, Japon

\section{${ }^{\star}$ Correspondance :} thirama@icloud.com

Citation proposée : Hirama T, Sabur NF, Derkach P, McNamee J, Song H, Marras TK, Brode SK. Facteurs de risque associés à la tuberculose pharmacorésistante dans un centre de référence situé à Toronto (Ontario) au Canada : 2010 à 2016. Relevé des maladies transmissibles au Canada 2020;46(4):95-103.

https://doi.org/10.14745/ccdr.v46i04a05f

Mots-clés : tuberculose, Mycobacterium tuberculosis, épreuve de sensibilité aux médicaments, résistant à l'isoniazide, multirésistant

\section{Introduction}

La tuberculose (TB) pharmacorésistante représente une menace majeure pour la santé publique dans le monde. Les deux formes de TB pharmacorésistante les plus courantes et les plus importantes sur le plan clinique sont la TB monorésistante à I'isoniazide (R-INH) (résistante à l'INH) et la TB multirésistante (MR) (résistante à au moins l'INH et la rifampicine, RMP) $(1,2)$. 
La pharmacorésistance est déterminée à l'aide de méthodes génotypiques ou d'une épreuve de sensibilité phénotypique aux médicaments (ESM) par culture cellulaire, cette dernière étant considérée comme la norme par excellence (3). La détermination de la pharmacorésistance est essentielle pour éclairer le choix de médicaments antimycobactériens adaptés et pour prévenir tout accroissement de la pharmacorésistance. Cependant, il peut s'écouler plusieurs semaines avant que l'ESM phénotypique ne livre ses résultats, et tous les milieux cliniques n'effectuent pas systématiquement d'ESM moléculaire rapide. C'est pourquoi les cliniciens préfèrent généralement engager un traitement empirique contre la TB avant que les résultats de I'ESM phénotypique ne soient disponibles. Ils peuvent également, en fonction des facteurs de risque du patient en matière de pharmacorésistance, élargir le traitement empirique initial ou demander une ESM moléculaire rapide.

Peu d'études ont décrit les facteurs de risque associés à la pharmacorésistance au Canada. En Colombie-Britannique, de 1990 à 2001, l'âge, la naissance à l'étranger, l'origine ethnique, l'existence d'antécédents de traitement, la réalisation du diagnostic en dehors du Canada et certaines régions du pays de naissance étaient des facteurs associés à la pharmacorésistance (4). En Alberta, de 1982 à 2011, l'âge (moins de 65 ans), l'existence d'antécédents de traitement, l'arrivée au Canada entre 2002 et 2011 et l'émigration récente en provenance des Philippines et du Vietnam étaient des facteurs de risque associés à la TB-MR chez les personnes nées à l'étranger (5). Selon une étude de surveillance nationale, entre 1997 et 2008, l'âge, la naissance à l'étranger, l'existence d'antécédents de traitement et certaines régions épidémiologiques de naissance définies par l'Organisation mondiale de la santé étaient des facteurs associés à la pharmacorésistance à l'échelle nationale (1). Cependant, aucune étude ne s'est intéressée aux facteurs de risque associés à la pharmacorésistance en Ontario, une province où les immigrants présentent un profil caractéristique (6) et où le fardeau imputable aux cas de TB pharmacorésistante est le plus lourd au Canada $(7,8)$. II convient également de recueillir un plus grand nombre de données contemporaines, car les facteurs de risque peuvent varier à mesure que les tendances d'immigration changent et que les taux de TB pharmacorésistante, notamment la TB-MR, évoluent dans le monde entier.

Cette étude avait pour objectif principal d'évaluer les facteurs de risque démographiques et cliniques potentiellement associés à la TB pharmacorésistante chez les patients traités au Centre de santé West Park (CSWP) et de comparer les facteurs de risque associés à la TB R-INH à ceux associés à la TB-MR. Par ailleurs, les patients tuberculeux participant à l'étude ont été évalués conformément aux dernières lignes directrices de l'American Thoracic Society, de I'Infectious Diseases Society of America et des Centers for Disease Control and Prevention (ATS/IDSA/ CDC) (3). Celles-ci recommandent que des analyses moléculaires rapides visant à déterminer la résistance à la RMP avec ou sans la résistance à l'INH soient menées chez les sous-groupes de patients suivants : 1) patients ayant des antécédents de traitement; 2) patients nés ou ayant vécu pendant un an ou plus dans un pays dont l'incidence de TB est égale ou supérieure à 20 cas pour 100000 personnes ou dont la prévalence de la MR primaire est égale ou supérieure à $2 \%$; 3 ) patients entrés en contact avec une personne atteinte de TB-MR; et 4) patients infectés par le virus de l'immunodéficience humaine $(\mathrm{VIH})$.

\section{Méthodes}

Situé à Toronto, en Ontario, le CSWP est un centre de référence pour la prise en charge de la TB pharmacorésistante, et traite la majorité des cas de TB-MR de la province ( $84 \%$ entre 2000 et 2011) (9). Tous les patients chez qui on a diagnostiqué la TB et qui ont été traités au CSWP entre janvier 2010 et décembre 2016 ont été pris en compte dans cette étude de cohorte rétrospective. L'examen des dossiers médicaux a permis de répertorier les patients à inclure dans l'étude ainsi qu'à extraire leurs caractéristiques démographiques et cliniques. Le protocole d'étude a été validé par le Comité conjoint d'éthique de la recherche Bridgepoint/West Park. Au regard du caractère rétrospectif de l'étude, l'obligation d'obtenir le consentement éclairé des patients a été levée.

Au cours de la période d'étude, toutes les épreuves de sensibilité aux médicaments ont été systématiquement effectuées au Laboratoire de tuberculose et de mycobactériologie de Santé publique Ontario (Toronto, Ontario). L'ESM a été réalisée selon les normes d'essai et les méthodes recommandées (le cas échéant) par le Clinical Laboratory Standards Institute, à l'aide d'un bouillon radiométrique [BACTEC 460; Becton, Dickinson et Co., Franklin Lakes, New Jersey, États-Unis (É.-U.)] jusqu'au 1er octobre 2010, et un bouillon non radiométrique (MGIT 960; Becton, Dickinson et Co.) par la suite $(10,11)$. La première culture du complexe Mycobacterium tuberculosis isolé auprès d'un patient a été systématiquement soumise à une épreuve de sensibilité aux quatre médicaments de première intention : INH, RMP, éthambutol et pyrazinamide. Les isolats capables de résister à l'INH à $0,1 \mathrm{mg} / \mathrm{l}$ étaient considérés comme "résistants» dans le cadre de cette étude, mais ont également été évalués à $0,4 \mathrm{mg} / \mathrm{l}$ et soumis à des essais associés à la moxifloxacine. Tout isolat qui s'est révélé résistant à la RMP ou à l'un des deux médicaments de première intention a été soumis à une ESM aux médicaments de deuxième intention. L'épreuve de sensibilité aux médicaments de deuxième intention énumérés ci-après a été réalisée au cours de la période d'étude : rifabutine, amikacine, streptomycine, kanamycine, capréomycine, ofloxacine, éthionamide et acide para-aminosalicylique. L'ESM relative à la clofazimine a été effectuée jusqu'au 1er octobre 2010, et l'ESM relative à la moxifloxacine a commencé le 1er octobre 2010.

Les caractéristiques des patients atteints d'une TB R-INH et d'une TB-MR ont été comparées avec celles des patients atteints d'une TB pharmacosensible (PS) (c'est-à-dire sensible aux quatre médicaments de première intention), à l'aide de modèles 
de régression logistique bivariée et multivariée. Les analyses statistiques ont été réalisées avec GraphPad Prism 6.0 (GraphPad Software; La Jolla, Californie, É.-U.), StatPlus:macLE (AnalystSoft; Walnut, Californie, É.-U.) et Jamovi (version 0.9 récupérée depuis https://www.jamovi.org). Dans les analyses bivariées, de nombreuses caractéristiques démographiques (âge, sexe, région du pays de naissance et temps écoulé depuis l'arrivée au Canada) et cliniques (facteurs de risque connus associés à la $T B$, localisation de la tuberculose et résultats microbiologiques) ont été analysées en vue de préciser leur éventuelle association avec la TB pharmacorésistante, un lien qui devra être étudié de manière plus approfondie. Les variables dont la valeur de $p$ est inférieure à 0,05 dans l'analyse bivariée, et les variables a priori considérées comme importantes sur le plan clinique (âge, sexe, région du pays de naissance, temps passé depuis l'arrivée au Canada et existence d'antécédents de traitement de la TB) ont été sélectionnées en vue de leur inclusion dans les modèles multivariés. Les modèles multivariés étaient limités aux patients nés à l'étranger de manière à ce que l'association entre le temps passé au Canada et la pharmacorésistance puisse être étudiée avec précision. Les patients ont également été répartis en groupes légèrement différents par l'ESM (pharmacosensibles, pharmacorésistance non multirésistante et MR/résistance à la RMP) pour évaluer les dernières recommandations des organismes ATS/IDSA/CDC concernant la réalisation d'une ESM moléculaire rapide relative à la rifampicine (3).

\section{Résultats}

Entre 2010 et 2016, 485 patients atteints d'une TB active ont été traités au CSWP, soit $11,1 \%$ des 4384 personnes traitées au total en Ontario (12). Parmi les patients du CSWP, les résultats de l'ESM étaient disponibles pour $82,9 \%$ d'entre eux $(n=402 / 485)$ (tableau 1). Pour les 83 autres patients $(17,1 \%)$, I'ESM phénotypique n'a pas pu être effectué en Ontario (en raison de l'absence de confirmation de culture ou d'un diagnostic établi en dehors de l'Ontario). Ces patients ont donc dû être exclus des prochaines analyses des facteurs de risque. Les souches tuberculeuses sensibles aux quatre médicaments de première intention représentaient $76,1 \%(n=306 / 402)$, les souches R-INH représentaient $10,9 \%(n=44 / 402)$ et les souches résistantes à l'INH, à la RMP et à d'autres médicaments (MR) représentaient $11,4 \%(n=46 / 402)$. Seuls quatre patients présentaient une monorésistance à des médicaments autres que l'INH (un à la RMP et trois à la PZA), deux présentaient une polyrésistance aux médicaments de première intention (mais pas de MR), et six patients ont été exclus des analyses de facteurs de risque. La TB ultrarésistante (MR caractérisée par une résistance supplémentaire à une fluoroquinolone et une préparation injectable de deuxième intention) était également rare, avec $1 \%$ $(n=4 / 402)$.

Les patients tuberculeux ont été répartis en trois groupes en fonction des résultats de l'ESM : TB-PS $(n=306), \mathrm{R}-\mathrm{INH}(n=44)$
Tableau 1 : Résultats de l'épreuve de sensibilité phénotypique aux médicaments chez les patients participants à l'étude

\section{Sensibilité aux médicaments $\quad n / N \quad n$}

Épreuve de sensibilité aux médicaments de première intention

( $n=402$ avec ESM disponible)

Sensibilité aux quatre médicaments

de première intention

Monorésistance à l'INH

Monorésistance à la RMP

B

Monorésistance à l'EMB

Monorésistance à la PZA

Polyrésistance aux médicaments de

première intention

Multirésistance (INH et RMP)

Ultrarésistance

Toute résistance à l'INH

Toute résistance à la RMP

Toute résistance à l'EMB

Toute résistance à la PZA

4

$306 / 402$

$44 / 402$

$1 / 402$

$0 / 402$

$3 / 402$

$\%$

Épreuve de sensibilité aux médicaments de deuxième intention $(n=46$ avec TB-MR)

\begin{tabular}{|l|r|r|}
\hline Toute résistance à l'EMB & $20 / 46$ & 43,5 \\
\hline Toute résistance à la PZA & $20 / 46$ & 43,5 \\
\hline Toute résistance à la RFB & $41 / 46$ & 89,1 \\
\hline Toute résistance à l'AMK & $3 / 45$ & 6,7 \\
\hline Toute résistance à la SM & $29 / 46$ & 63,0 \\
\hline Toute résistance à la KM & $6 / 41$ & 14,6 \\
\hline Toute résistance à la CM & $6 / 46$ & 13,0 \\
\hline Toute résistance à la MFX & $5 / 41$ & 12,2 \\
\hline Toute résistance à l'OFX & $8 / 46$ & 17,4 \\
\hline Toute résistance à l'ETA & $13 / 46$ & 28,3 \\
\hline Toute résistance au PAS & $5 / 46$ & 10,9 \\
\hline Toute résistance au LZD & $0 / 41$ & 0,0 \\
\hline Toute résistance à la CLO & $0 / 5$ & 0,0 \\
\hline
\end{tabular}

Abréviations : AMK, amikacine; CLO, clofazimine; CM, capréomycine; EMB, éthambutol; $E S M$, épreuve de sensibilité aux médicaments; ETA, éthionamide; $I N H$, isoniazide;

$\mathrm{KM}$, kanamycine; LZD, linézolide; MR, multirésistant; MXF, moxifloxacine; OFX, ofloxacine;

KM, kanamycine; LZD, linezolide; MR, multiresistant; MXF, moxifloxacine; OFX, ofloxacine;
PAS, acide para-aminosalicylique; PZA, pyrazinamide; RFB, rifabutine; SM, streptomycine; TB, tuberculose

et MR $(n=46)$. Leurs caractéristiques démographiques sont présentées au tableau 2 et leurs caractéristiques cliniques au tableau 3. Par rapport aux patients atteints d'une TB-PS (tableau 2), dans les analyses non rajustées, les patients atteints d'une TB R-INH étaient beaucoup plus jeunes (rapport de cotes [RC] pour les personnes de moins de 35 ans $=2,58$, IC de $95 \%$ allant de 1,06 à 6,30), pour un âge de référence supérieur à 65 ans, et moins susceptibles d'être nés en Asie du Sud-Est (RC de 0,157, IC de $95 \%$ allant de 0,03 à 0,91). Les patients atteints d'une TB R-INH (tableau 3) étaient également plus susceptibles de présenter des antécédents de traitement (RC de 
Tableau 2 : Caractéristiques démographiques des patients tuberculeux participant à l'étude

\begin{tabular}{|c|c|c|c|c|c|c|c|c|c|c|c|c|c|c|}
\hline \multirow{2}{*}{$\begin{array}{l}\text { Caractéristiques } \\
\text { démographiques }\end{array}$} & \multicolumn{2}{|c|}{$\begin{array}{c}\text { Tous les } \\
\text { patients } \\
\text { tuberculeux }\end{array}$} & \multicolumn{2}{|c|}{ TB-PS } & \multicolumn{2}{|c|}{ TB R-INH } & \multicolumn{3}{|c|}{ TB R-INH ou TB-PS } & \multicolumn{2}{|c|}{ TB-MR } & \multicolumn{3}{|c|}{ TB-MR ou TB-PS } \\
\hline & $(n=485)$ & $\%$ & $(n=306)$ & $\%$ & $(n=44)$ & $\%$ & $\mathrm{RC}$ & $\begin{array}{c}\text { (IC de } 95 \\
\%)\end{array}$ & $\begin{array}{l}\text { Valeur } \\
\text { de } p\end{array}$ & $(n=46)$ & $\%$ & $\mathrm{RC}$ & (IC de $95 \%)$ & $\begin{array}{l}\text { Valeur } \\
\text { de } p\end{array}$ \\
\hline \multicolumn{15}{|l|}{ Âge, en années } \\
\hline Moins de 35 ans & 146 & 30,1 & 79 & 25,8 & 17 & 38,6 & 2,58 & $\begin{array}{r}(1,06 \text { à } \\
6,30)\end{array}$ & 0,037 & 25 & 54,3 & 15,1 & $\begin{array}{r}(3,49 \text { à } \\
66,11)\end{array}$ & 0,01 \\
\hline 35 à 65 ans & 212 & 43,7 & 131 & 42,8 & 19 & 43,2 & 1,74 & $\begin{array}{r}(0,73 \text { à } \\
4,14)\end{array}$ & 0,210 & 19 & 41,3 & 6,96 & $(1,58$ à 30,6$)$ & $<0,001$ \\
\hline Plus de 65 ans & 127 & 26,2 & 96 & 31,4 & 8 & 18,2 & 1,0 & référence ${ }^{a}$ & $\mathrm{~S} / \mathrm{O}$ & 2 & 4,3 & 1,0 & référence ${ }^{\mathrm{a}}$ & $\mathrm{S} / \mathrm{O}$ \\
\hline \multicolumn{15}{|l|}{ Genre } \\
\hline Sexe, femme & 215 & 44,3 & 120 & 39,2 & 24 & 54,5 & 1,86 & $\begin{array}{r}(0,99 \text { à } \\
3,51)\end{array}$ & 0,056 & 24 & 52,2 & 1,69 & $(0,91$ à 3,15$)$ & 0,098 \\
\hline \multicolumn{15}{|l|}{ Pays de naissance } \\
\hline Né à l'étranger & 450 & 92,8 & 280 & 91,5 & 40 & 90,9 & 0,93 & $\begin{array}{r}(0,31 \text { à } \\
2,80) \\
\end{array}$ & 0,895 & 45 & 97,8 & 4,18 & $\begin{array}{r}(0,56 \text { à } \\
31,53)\end{array}$ & 0,166 \\
\hline Né au Canada & 35 & 7,2 & 26 & 8,5 & 4 & 9,1 & 1,0 & référence ${ }^{\mathrm{a}}$ & $\mathrm{S} / \mathrm{O}$ & 1 & 2,2 & 1,0 & référence ${ }^{a}$ & $\mathrm{~S} / \mathrm{O}$ \\
\hline \multicolumn{15}{|c|}{ Région du pays de naissance définie par l'OMS } \\
\hline Région africaine & 43 & 8,9 & 27 & 8,8 & 3 & 6,8 & 0,72 & $\begin{array}{r}(0,15 \text { à } \\
3,54)\end{array}$ & 0,688 & 2 & 4,3 & 1,92 & $(0,16$ à 22,5$)$ & 0,602 \\
\hline $\begin{array}{l}\text { Région des } \\
\text { Amériques }^{b}\end{array}$ & 27 & 5,6 & 18 & 5,9 & 3 & 6,8 & 1,08 & $\begin{array}{r}(0,22 \text { à } \\
5,44)\end{array}$ & 0,923 & 0 & 0,0 & $\mathrm{~S} / \mathrm{O}$ & référence ${ }^{\mathrm{a}}$ & $\mathrm{S} / \mathrm{O}$ \\
\hline $\begin{array}{l}\text { Région de la } \\
\text { Méditerranée } \\
\text { orientale }\end{array}$ & 49 & 10,1 & 29 & 9,5 & 5 & 11,4 & 1,12 & $\begin{array}{r}(0,27 \text { à } \\
4,62)\end{array}$ & 0,875 & 2 & 4,3 & 1,79 & $(0,15$ à 20,9$)$ & 0,641 \\
\hline $\begin{array}{l}\text { Région } \\
\text { européenne }\end{array}$ & 21 & 4,3 & 10 & 3,3 & 0 & 0,0 & 1,0 & référence ${ }^{\mathrm{a}}$ & $\mathrm{S} / \mathrm{O}$ & 6 & 13,0 & 15,6 & $\begin{array}{r}(1,66 \text { à } \\
146,4)\end{array}$ & 0,016 \\
\hline $\begin{array}{l}\text { Région de l'Asie } \\
\text { du Sud-Est }\end{array}$ & 124 & 25,6 & 83 & 27,1 & 2 & 4,5 & 0,157 & $\begin{array}{r}(0,03 \text { à } \\
0,91) \\
\end{array}$ & 0,038 & 14 & 30,4 & 4,38 & $(0,55$ à 34,9$)$ & 0,163 \\
\hline $\begin{array}{l}\text { Région du } \\
\text { Pacifique } \\
\text { occidental }\end{array}$ & 186 & 38,4 & 113 & 36,9 & 27 & 61,4 & 1,55 & $\begin{array}{r}(0,50 \text { à } \\
4,82)\end{array}$ & 0,446 & 21 & 45,7 & 4,83 & $(0,62$ à 37,5$)$ & 0,132 \\
\hline Canada & 35 & 7,2 & 26 & 8,5 & 4 & 9,1 & 1,0 & référence ${ }^{\mathrm{a}}$ & $\mathrm{S} / \mathrm{O}$ & 1 & 2,2 & 1,0 & référence ${ }^{a}$ & $\mathrm{~S} / \mathrm{O}$ \\
\hline \multicolumn{15}{|c|}{ Nombre de mois passés entre l'arrivée et le diagnostic de TB ${ }^{c}$} \\
\hline Moins de 24 mois & 93 & 19,2 & 45 & 14,7 & 6 & 13,6 & 1,13 & $\begin{array}{r}(0,42 \text { à } \\
3,01)\end{array}$ & 0,813 & 18 & 39,1 & 7,60 & $(3,09$ à 18,6$)$ & $<0,001$ \\
\hline 24 à 120 mois & 141 & 29,0 & 80 & 26,1 & 16 & 36,4 & 1,69 & $\begin{array}{r}(0,82 \text { à } \\
3,50)\end{array}$ & 0,157 & 19 & 41,3 & 4,51 & $(1,89$ à 10,7$)$ & $<0,001$ \\
\hline Plus de 120 mois & 213 & 43,9 & 152 & 49,7 & 18 & 40,9 & 1,0 & référence ${ }^{\mathrm{a}}$ & $\mathrm{S} / \mathrm{O}$ & 8 & 17,4 & 1,0 & référence ${ }^{a}$ & $\mathrm{~S} / \mathrm{O}$ \\
\hline
\end{tabular}

Abréviations : MR, multirésistant; OMS, Organisation mondiale de la santé; PS, pharmacosensible; R-INH, monorésistant à l'isoniazide; $\mathrm{S} / \mathrm{O}$, sans objet; TB, tuberculose

a "Référence» s'entend du groupe de contrôle auquel tous les autres groupes sont comparés

'À l'exception du Canada

c Patients nés à l'étranger uniquement; date d'arrivée manquante pour trois patients 
Tableau 3 : Caractéristiques cliniques des patients tuberculeux participant à l'étude

\begin{tabular}{|c|c|c|c|c|c|c|c|c|c|c|c|c|c|c|}
\hline \multirow{2}{*}{$\begin{array}{l}\text { Caractéristiques } \\
\text { cliniques }\end{array}$} & \multicolumn{2}{|c|}{$\begin{array}{c}\text { Tous les } \\
\text { patients } \\
\text { tuberculeux }\end{array}$} & \multicolumn{2}{|c|}{ TB-PS } & \multicolumn{2}{|c|}{ TB R-INH } & \multicolumn{3}{|c|}{ TB R-INH ou TB-PS } & \multicolumn{2}{|c|}{ TB-MR } & \multicolumn{3}{|c|}{ TB-MR ou TB-PS } \\
\hline & $(n=485)$ & $\%$ & $(n=306)$ & $\%$ & $(n=44)$ & $\%$ & $\mathrm{RC}$ & (IC de $95 \%)$ & $\begin{array}{l}\text { Valeur } \\
\text { de } p\end{array}$ & $(n=46)$ & $\%$ & $\mathrm{RC}$ & (IC de $95 \%)$ & $\begin{array}{l}\text { Valeur } \\
\text { de } p\end{array}$ \\
\hline \multicolumn{15}{|c|}{ Facteur de risque associé à la TB } \\
\hline $\begin{array}{l}\text { Existence } \\
\text { d'antécédents de } \\
\text { traitement de la TB }\end{array}$ & 70 & 14,4 & 26 & 8,5 & 8 & 18,2 & 2,39 & $(1,01$ à 5,68$)$ & 0,048 & 16 & 34,8 & 5,74 & $(2,77$ à 11,89$)$ & $<0,001$ \\
\hline $\begin{array}{l}\text { Antécédents de } \\
\text { contacts avec une } \\
\text { personne atteinte } \\
\text { de TB }\end{array}$ & 102 & 21,0 & 64 & 20,9 & 7 & 15,9 & 0,72 & $(0,31$ à 1,6$)$ & 0,442 & 9 & 19,6 & 0,92 & $(0,42$ à 2,0$)$ & 0,833 \\
\hline $\begin{array}{l}\text { Antécédents de } \\
\text { contacts avec } \\
\text { une personne } \\
\text { atteinte ou } \\
\text { soupçonnée d'être } \\
\text { atteinte de TB } \\
\text { pharmacorésistante }\end{array}$ & 4 & 0,8 & 1 & 0,3 & 1 & 2,3 & 7,09 & $(0,44$ à 115,5$)$ & 0,169 & 1 & 2,2 & 6,77 & $(0,41$ à 111,2$)$ & 0,179 \\
\hline $\begin{array}{l}\text { Voyage vers } \\
\text { une région où } \\
\text { l'incidence est } \\
\text { élevée }\end{array}$ & 165 & 34,0 & 105 & 34,3 & 13 & 29,5 & 0,80 & $(0,40$ à 1,60$)$ & 0,532 & 14 & 30,4 & 0,84 & $(0,42$ à 1,63$)$ & 0,604 \\
\hline $\begin{array}{l}\text { Séjour dans un } \\
\text { camp de réfugiés }\end{array}$ & 26 & 5,4 & 19 & 6,2 & 0 & 0,0 & $\mathrm{~S} / \mathrm{O}$ & $\mathrm{S} / \mathrm{O}$ & $\mathrm{S} / \mathrm{O}$ & 2 & 4,3 & 0,65 & $(0,14$ à 2,87$)$ & 0,57 \\
\hline $\begin{array}{l}\text { Sans-abri ou } \\
\text { incarcéré }\end{array}$ & 44 & 9,1 & 33 & 10,8 & 3 & 6,8 & 0,61 & $(0,18$ à 2,96$)$ & 0,422 & 2 & 4,3 & 0,37 & $(0,08$ à 1,62$)$ & 0,19 \\
\hline $\begin{array}{l}\text { Consommation de } \\
\text { drogues illicites }\end{array}$ & 31 & 6,4 & 22 & 7,2 & 6 & 13,6 & 2,04 & $(0,78$ à 5,35$)$ & 0,148 & 1 & 2,2 & 0,28 & $(0,03$ à 2,18$)$ & 0,287 \\
\hline $\begin{array}{l}\text { Consommation } \\
\text { régulière d'alcool }\end{array}$ & 172 & 35,5 & 117 & 38,2 & 13 & 29,5 & 0,68 & $(0,34$ à 1,35$)$ & 0,267 & 17 & 37,0 & 0,95 & $(0,49$ à 1,79$)$ & 0,868 \\
\hline $\begin{array}{l}\text { Consommation de } \\
\text { tabac (actuelle ou } \\
\text { antérieure) }\end{array}$ & 145 & 29,9 & 98 & 32,0 & 15 & 34,1 & 1,10 & $(0,56$ à 2,14$)$ & 0,784 & 16 & 34,8 & 1,13 & $(0,58$ à 2,17$)$ & 0,71 \\
\hline Malignité active & 21 & 4,3 & 12 & 3,9 & 1 & 2,3 & 0,57 & $(0,07$ à 4,50$)$ & 0,593 & 3 & 6,5 & 1,71 & $(0,46$ à 6,30$)$ & 0,421 \\
\hline $\begin{array}{l}\text { Traitement } \\
\text { immunosuppresseur }\end{array}$ & 14 & 2,9 & 9 & 2,9 & 1 & 2,3 & 0,77 & $(0,95$ à 6,21$)$ & 0,804 & 2 & 4,3 & 1,5 & $(0,031$ à 7,17$)$ & 0,611 \\
\hline Diabète & 85 & 17,5 & 67 & 21,9 & 3 & 6,8 & 0,26 & $(0,08$ à 0,87$)$ & 0,029 & 8 & 17,4 & 0,75 & $(0,33$ à 1,68$)$ & 0,488 \\
\hline Infection au VIH & 10 & 2,1 & 6 & 2,0 & 0 & 0,0 & $\mathrm{~S} / \mathrm{O}$ & $\mathrm{S} / \mathrm{O}$ & $\mathrm{S} / \mathrm{O}$ & 3 & 6,5 & 4,76 & $(1,29$ à 17,5$)$ & 0,019 \\
\hline \multicolumn{15}{|l|}{ Répartition des TB } \\
\hline $\begin{array}{l}\text { TB pulmonaire } \\
\text { uniquement }\end{array}$ & 280 & 57,7 & 176 & 57,5 & 28 & 63,6 & 1,29 & $(0,67$ à 2,49$)$ & 0,442 & 34 & 73,9 & 2,09 & $(1,04$ à 4,19$)$ & 0,038 \\
\hline $\begin{array}{l}\text { TB pulmonaire et } \\
\text { extrapulmonaire }\end{array}$ & 103 & 21,2 & 75 & 24,5 & 11 & 25,0 & 1,02 & $(0,49$ à 2,13$)$ & 0,944 & 5 & 10,9 & 0,37 & $(0,14$ à 0,98$)$ & 0,047 \\
\hline $\begin{array}{l}\text { TB extrapulmonaire } \\
\text { uniquement }\end{array}$ & 102 & 21,0 & 55 & 18,0 & 5 & 11,4 & 0,59 & $(0,22$ à 1,55$)$ & 0,282 & 7 & 15,2 & 0,82 & $(0,34$ à 1,92$)$ & 0,648 \\
\hline $\begin{array}{l}\text { Caverne détectée } \\
\text { par radiographie } \\
\text { thoracique }\end{array}$ & 98 & 20,2 & 72 & 23,5 & 9 & 20,5 & 0,84 & $(0,38$ à 1,82$)$ & 0,651 & 9 & 19,6 & 0,79 & $(0,36$ à 1,71$)$ & 0,552 \\
\hline \multicolumn{15}{|c|}{ Analyse des bacilles acidorésistants } \\
\hline $\begin{array}{l}\text { Frottis positif } \\
\text { aux bacilles } \\
\text { acidorésistants dans } \\
\text { le crachat }\end{array}$ & 191 & 39,4 & 155 & 50,7 & 25 & 56,8 & 1,60 & $(0,80$ à 3,20$)$ & 0,179 & 17 & 37,0 & 0,57 & $(0,29$ à 1,08$)$ & 0,089 \\
\hline
\end{tabular}


2,39, IC de $95 \%$ allant de 1,01 à 5,68), et moins susceptibles de souffrir de diabète (RC de $0,26, I C$ de $95 \%$ allant de 0,08 à 0,87 ) dans les analyses non rajustées. Par rapport aux patients atteints d'une TB-PS, dans les analyses non rajustées, les patients atteints d'une TB-MR (tableau 2) étaient considérablement plus jeunes (moins de 35 ans, RC de 15,2, IC de $95 \%$ allant de 3,49 à 66,1), plus susceptibles d'être nés en Europe (RC de 15,6, IC de $95 \%$ allant de 1,66 à 146,4) et affichaient une durée beaucoup plus courte entre leur arrivée au Canada et le diagnostic de la TB. Ces patients (tableau 3 ) étaient plus susceptibles de présenter des antécédents de traitement (RC de 5,74, IC de $95 \%$ allant de 2,77 à 11,9), plus susceptibles d'être infectés par le VIH (RC de 4,76, IC de $95 \%$ allant de 1,29 à 17,5), plus susceptibles de n'être touchés que par une TB pulmonaire, et moins susceptibles d'être atteints d'une TB pulmonaire et extrapulmonaire.
Dans l'analyse multivariée limitée aux patients nés à l'étranger (tableau 4), les patients atteints de TB R-INH étaient moins susceptibles d'être originaires de l'Asie du Sud-Est que les patients atteints de TB-PS (RC de 0,10, IC de $95 \%$ allant de 0,01 à 0,73 ). Ils étaient également moins susceptibles d'être diabétiques (RC de 0,18, IC de $95 \%$ allant de 0,04 à 0,81). Les facteurs de risque associés à la TB-MR dans l'analyse multivariée limitée aux patients nés à l'étranger étaient l'appartenance à un groupe d'âge plus jeune (moins de 35 ans) (RC de 8,11, IC de $95 \%$ allant de 1,43 à 45,7), l'établissement d'un diagnostic de TB moins de 24 mois après l'arrivée au Canada (RC de 4,11, IC de $95 \%$ allant de 1,21 à 13,9), l'existence d'antécédents de traitement de la TB (RC de 3,78, IC de $95 \%$ allant de 1,58 à 9,05) et l'infection par le VIH (RC de 10,95, IC de $95 \%$ allant de 1,90 à $62,9)$.

\section{Tableau 4 : Facteurs de risque associés à la tuberculose monorésistante à l'isoniazide et à la tuberculose multirésistante chez les patients nés à l'étranger traités au Centre de santé West Park}

\begin{tabular}{|c|c|c|c|c|c|c|}
\hline \multirow{2}{*}{ Facteurs de risque } & \multicolumn{3}{|c|}{ TB R-INH ou TB-PS } & \multicolumn{3}{|c|}{ TB-MR ou TB-PS } \\
\hline & $\mathrm{RC}$ & (IC de $95 \%)$ & Valeur de $p$ & $\mathrm{RC}$ & (IC de $95 \%$ ) & Valeur de $p$ \\
\hline \multicolumn{7}{|l|}{ Âge, en années } \\
\hline Moins de 35 ans & 1,69 & $(0,54$ à 5,26$)$ & 0,365 & 8,11 & $(1,43$ à 45,7$)$ & 0,018 \\
\hline 35 à 65 ans & 1,16 & $(0,44$ à 3,06$)$ & 0,76 & 4,84 & $(0,94$ à 24,7$)$ & 0,058 \\
\hline Plus de 65 ans & 1,0 & référence ${ }^{a}$ & $\mathrm{~S} / \mathrm{O}$ & 1,0 & référence ${ }^{a}$ & $\mathrm{~S} / \mathrm{O}$ \\
\hline \multicolumn{7}{|l|}{ Genre } \\
\hline Sexe, femme & 1,36 & $(0,65$ à 2,88$)$ & 0,408 & 1,57 & $(0,71$ à 3,47$)$ & 0,265 \\
\hline \multicolumn{7}{|c|}{ Région du pays de naissance définie par l'OMS } \\
\hline Région africaine & 0,46 & $(0,08$ à 2,64$)$ & 0,384 & 0,45 & $(0,05$ à 3,86$)$ & 0,468 \\
\hline Région des Amériques & 1,0 & référence ${ }^{a}$ & $\mathrm{~S} / \mathrm{O}$ & $\mathrm{S} / \mathrm{O}$ & $\mathrm{S} / \mathrm{O}$ & $\mathrm{S} / \mathrm{O}$ \\
\hline $\begin{array}{l}\text { Région de la Méditerranée } \\
\text { orientale }^{\mathrm{b}}\end{array}$ & 0,90 & $(0,17$ à 4,81$)$ & 0,909 & 1,0 & référence ${ }^{a}$ & $\mathrm{~S} / \mathrm{O}$ \\
\hline Région européenne & $\mathrm{S} / \mathrm{O}$ & $\mathrm{S} / \mathrm{O}$ & $\mathrm{S} / \mathrm{O}$ & 4,29 & $(0,54$ à 33,7$)$ & 0,166 \\
\hline Région de l'Asie du Sud-Est & 0,10 & $(0,01$ à 0,73$)$ & 0,023 & 1,31 & $(0,25$ à 6,95$)$ & 0,744 \\
\hline Région du Pacifique occidental & 1,50 & $(0,38$ à 5,83$)$ & 0,558 & 1,97 & $(0,38$ à 10,2$)$ & 0,415 \\
\hline
\end{tabular}

Nombre médian de mois passés entre l'arrivée et le diagnostic de TB

\begin{tabular}{|c|c|c|c|c|c|c|}
\hline Moins de 24 mois & 1,10 & $(0,34$ à 3,52$)$ & 0,861 & 4,11 & $(1,21$ à 13,9$)$ & 0,023 \\
\hline 24 à 120 mois & 1,26 & $(0,53$ à 3,0$)$ & 0,588 & 2,48 & $(0,83$ à 7,35$)$ & 0,101 \\
\hline Plus de 120 mois & 1,0 & référence ${ }^{a}$ & $\mathrm{~S} / \mathrm{O}$ & 1,0 & référence ${ }^{a}$ & $\mathrm{~S} / \mathrm{O}$ \\
\hline \multicolumn{7}{|l|}{ Facteur de risque associé à la TB } \\
\hline $\begin{array}{l}\text { Existence d'antécédents de } \\
\text { traitement de la TB }\end{array}$ & 2,21 & $(0,73$ à 6,15$)$ & 0,163 & 3,78 & $(1,58$ à 9,05$)$ & 0,003 \\
\hline Diabète & 0,18 & $(0,04$ à 0,81$)$ & 0,026 & $\mathrm{~S} / \mathrm{O}$ & $\mathrm{S} / \mathrm{O}$ & $\mathrm{S} / \mathrm{O}$ \\
\hline Infection au VIH & $\mathrm{S} / \mathrm{O}$ & $\mathrm{S} / \mathrm{O}$ & $\mathrm{S} / \mathrm{O}$ & 10,95 & $(1,90$ à 62,9$)$ & 0,007 \\
\hline \multicolumn{7}{|l|}{ Répartition des TB } \\
\hline TB pulmonaire uniquement & $\mathrm{S} / \mathrm{O}$ & $\mathrm{S} / \mathrm{O}$ & $\mathrm{S} / \mathrm{O}$ & 2,76 & $(0,92$ à 8,19$)$ & 0,067 \\
\hline TB pulmonaire et extrapulmonaire & $\mathrm{S} / \mathrm{O}$ & $\mathrm{S} / \mathrm{O}$ & $\mathrm{S} / \mathrm{O}$ & 0,70 & $(0,17$ à 2,77$)$ & 0,617 \\
\hline
\end{tabular}

Abréviations : MR, multirésistant; OMS, Organisation mondiale de la santé; PS, pharmacosensible; $\mathrm{R}-\mathrm{INH}$, monorésistant à l'isoniazide; $\mathrm{S} / \mathrm{O}$, sans objet; TB, tuberculose; VIH, virus de l'immunodéficience humaine

a "Référence» s'entend du groupe de contrôle auquel tous les autres groupes sont comparés

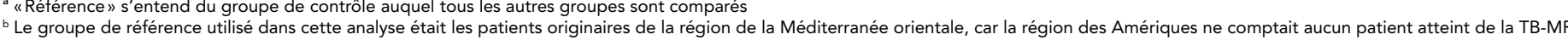

' La date d'arrivée est manquante pour trois patients 
Dans notre évaluation des recommandations présentées en 2017 par les organismes ATS/IDSA/CDC concernant la réalisation d'une ESM moléculaire rapide relative à la rifampicine, nous avons constaté que les patients qui présentaient une multirésistance ou une résistance à la RMP étaient largement plus susceptibles d'avoir des antécédents de traitement de la TB (RC de 5,39, IC de $95 \%$ allant de 2,57 à 11,3) et d'être infectés par le VIH (RC de 4,26, IC de $95 \%$ allant de 1,06 à 17,0) que les patients atteints de TB-PS dans l'analyse multivariée (tableau 5).

\section{Discussion}

Nous avons recensé sept facteurs de risque associés à la pharmacorésistance chez les patients tuberculeux traités dans notre établissement de Toronto, en Ontario. Pour ce qui est de la TB R-INH, nous avons constaté que l'appartenance à un groupe d'âge jeune (moins de 35 ans), l'existence d'antécédents de traitement de la TB, l'absence de diabète et la naissance dans un pays situé ailleurs qu'en Asie du Sud-Est constituaient des facteurs de risque dans l'analyse bivariée (non rajustée), mais que seuls les deux derniers facteurs étaient déterminants dans l'analyse multivariée. L'existence d'antécédents de traitement de la TB avait déjà été signalée comme étant un facteur de risque associé à la TB R-INH, même après ajustement des variables confusionnelles (13). C'est avec une certaine surprise que nous avons mis en évidence un lien significatif entre le diabète et la TB R-INH dans notre analyse bivariée et multivariée, le premier semblant jouer un rôle "protecteur» contre la dernière. La plupart des études précédentes n'ont pas pris en compte le diabète dans l'évaluation des facteurs de risque associés à la pharmacorésistance, et une étude menée en Colombie-Britannique n'a établi aucune corrélation entre ces deux variables (4). Par ailleurs, certains rapports, notamment une méta-analyse récente, ont fait état d'une corrélation positive entre le diabète et la TB-MR (14). Étant donné qu'aucune étude n'a démontré de corrélation négative entre la TB R-INH et le diabète, et que ce constat ne repose sur aucune explication biologique plausible, nous croyons que cette corrélation pourrait être fausse. Bien que nous ayons tenu compte de l'âge dans notre modèle multivarié, il se peut que ce facteur ait induit une confusion résiduelle, car la TB R-INH était plus courante chez les patients plus jeunes, chez qui la prévalence du diabète est généralement moins élevée. Il se peut également que cette corrélation ait été découverte par hasard, et qu'elle soit liée à des comparaisons multiples; elle mérite de faire l'objet d'études complémentaires. II est intéressant de noter que la naissance à l'étranger ( $R C$ de 0,93, IC de $95 \%$ allant de 0,31 à 2,80) n'était pas associée à la TB R-INH dans la population étudiée. Même si d'autres études nord-américaines ont constaté que la naissance à l'étranger était un facteur de risque associé à la TB R-INH ou la TB monorésistante $(1,4,13)$, seule une de ces études a été soumise à un ajustement des variables confusionnelles et aucune corrélation n'a été établie (13).

Nous avons mis en évidence, dans la population étudiée, plusieurs facteurs de risque associés à la TB-MR qui avaient déjà été décrits en Amérique du Nord, notamment l'appartenance à une génération plus jeune, l'existence d'antécédents de traitement (5), l'arrivée plus récente au Canada (1) et l'infection au VIH. L'infection au VIH prête à controverse; une méta-analyse a montré que la plupart des études nord-américaines faisaient état d'une corrélation. Aucune corrélation significative avec la TB-MR n'a cependant été démontrée après avoir pris en compte les études réalisées dans toutes les régions du monde; une corrélation avec la TB-MR primaire a toutefois été observée (15). Dans l'analyse bivariée, nous avons également constaté que les patients atteints d'une TB pulmonaire uniquement étaient plus susceptibles de présenter une TB-MR, contrairement aux patients atteints d'une TB pulmonaire et extrapulmonaire. II est possible

Tableau 5 : Critères recommandés par l'ATS dans ses lignes directrices 2017 concernant la réalisation d'une épreuve de sensibilité moléculaire rapide relative à la rifampicine ${ }^{a}$

\begin{tabular}{|c|c|c|c|c|c|c|c|c|c|c|c|c|c|c|}
\hline \multirow[t]{2}{*}{ Critères } & \multicolumn{2}{|c|}{$\begin{array}{c}\text { Tous les } \\
\text { patients } \\
\text { tuberculeux }\end{array}$} & \multicolumn{2}{|c|}{ TB-PS } & \multicolumn{2}{|c|}{$\begin{array}{c}\text { TB non MR/ } \\
\text { pharmacorésistante } \\
\text { à la RMP }\end{array}$} & \multicolumn{2}{|c|}{$\begin{array}{c}\text { TB-MR/R à la } \\
\text { RMP }\end{array}$} & \multicolumn{3}{|c|}{$\begin{array}{c}\text { TB-MR/R à la RMP } \\
\text { ou TB-PS }\end{array}$} & \multicolumn{3}{|c|}{$\begin{array}{c}\text { TB-MR/R à la RMP ou TB non } \\
\text { MR/pharmacorésistante à la } \\
\text { RMP }\end{array}$} \\
\hline & $(n=485)$ & $\%$ & $(n=306)$ & $\%$ & $(n=49)$ & $\%$ & $(n=47)$ & $\%$ & $\mathrm{RC}$ & (IC de $95 \%$ ) & $\begin{array}{l}\text { Valeur } \\
\text { de } p\end{array}$ & $\mathrm{RC}$ & (IC de $95 \%)$ & $\begin{array}{l}\text { Valeur } \\
\text { de } p\end{array}$ \\
\hline $\begin{array}{l}\text { Existence } \\
\text { d'antécédents } \\
\text { de traitement } \\
\text { de la TB }\end{array}$ & 70 & 14,4 & 26 & 8,5 & 9 & 18,4 & 16 & 34,0 & 5,39 & $(2,57$ à 11,3$)$ & $<0,001$ & 2,21 & $(0,83$ à 5,90$)$ & 0,112 \\
\hline $\begin{array}{l}\text { Naissance } \\
\text { ou résidence } \\
\text { pendant moins } \\
\text { d'un an dans } \\
\text { un pays à } \\
\text { risque élevé }\end{array}$ & 428 & 88,2 & 264 & 86,3 & 43 & 87,8 & 45 & 95,7 & 2,72 & $(0,62$ à 11,2$)$ & 0,184 & 3,02 & $(0,56$ à 16,2$)$ & 0,197 \\
\hline $\begin{array}{l}\text { Contact avec } \\
\text { une personne } \\
\text { atteinte de } \\
\text { TB-MR }\end{array}$ & 4 & 0,8 & 1 & 0,3 & 2 & 4,1 & 1 & 2,1 & 9,04 & $(0,54$ à 148,7$)$ & 0,123 & 0,46 & $(0,03$ à 5,60$)$ & 0,548 \\
\hline $\begin{array}{l}\text { Infection au } \\
\text { VIH }\end{array}$ & 10 & 2,1 & 6 & 2,0 & 0 & 0,0 & 4 & 8,5 & 4,26 & $(1,06$ à 17,0$)$ & 0,04 & $\mathrm{~S} / \mathrm{O}$ & $\mathrm{S} / \mathrm{O}$ & $\mathrm{S} / \mathrm{O}$ \\
\hline
\end{tabular}

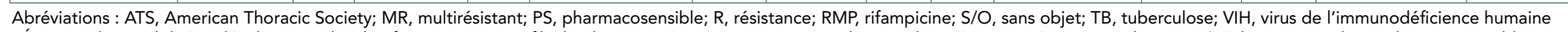

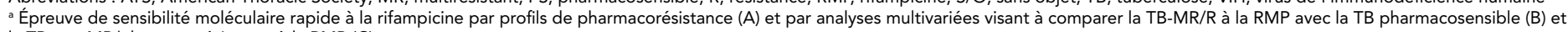
la TB non MR/pharmacorésistante à la RMP (C)

b Incidence de la TB supérieure ou égale à 20 cas pour 100000 personnes ou prévalence de la MR primaire égale ou supérieure à $2 \%$ 
que la répartition des TB ait été faussée par la date à laquelle leur diagnostic a été réalisé, car les patients atteints d'une TB-MR étaient plus susceptibles d'être arrivés récemment au Canada et, par conséquent, leur maladie pouvait se trouver à un stade moins avancé. En effet, la proportion globale de l'affection pulmonaire (pulmonaire et pulmonaire et extrapulmonaire) était similaire entre les catégories de l'ESM $(82,0 \%(n=251 / 306)$ pour la TB-PS; 88,6 \% $(n=39 / 44)$ pour la TB R-INH; 84,8 \% ( $n=$ 39/46) pour la TB-MR).

Concernant les régions de naissance, aucune corrélation significative n'est ressortie de l'analyse multivariée, mais la naissance en Europe (RC de 15,6 \%, IC de $95 \%$ allant de 1,66 à 146,4$)$ était un facteur de risque associé à la TB-MR dans l'analyse bivariée, et l'absence de signification dans l'analyse multivariée de notre étude pourrait s'expliquer par le faible nombre de cas analysés.

Vu le nombre croissant d'immigrants au Canada (5,5 millions en 2000 à 7,9 millions en 2017) (16,17) et l'épidémie de TB pharmacorésistante qui sévit dans le monde, la prévalence de la TB pharmacorésistante pourrait bien s'accroître au Canada (8). L'une des nombreuses difficultés que pose la TB pharmacorésistante dans les pays où le fardeau de la morbidité est faible est l'intervalle entre le moment où la TB est diagnostiquée et le moment où les résultats de l'ESM par culture cellulaire sont connus, ce qui risque d'allonger le temps avant de pouvoir commencer un traitement adapté, d'accroître la morbidité et d'entretenir l'infectiosité. Cependant, dans ces régions, les analyses moléculaires universelles rapides ne sont pas nécessairement rentables et peuvent générer un nombre élevé de faux positifs (18). C'est pourquoi les analyses ciblées, fondées sur les facteurs de risque, sont plus souvent utilisées. Les dernières lignes directrices des organismes ATS/IDSA/CDC relativement au diagnostic de la TB (3) recommandent que des analyses moléculaires rapides visant à déterminer la résistance à la RMP avec ou sans résistance à l'INH soient menées chez les sous-groupes de patients suivants : 1) patients ayant des antécédents de traitement; 2) patients nés ou ayant vécu pendant au moins un an dans un pays dont l'incidence de la TB est égale ou supérieure à 20 cas pour 100000 personnes, ou dont la prévalence de la MR primaire est égale ou supérieure à $2 \%$; 3 ) patients entrés en contact avec une personne atteinte de TB-MR; et 4) patients infectés par le VIH. Nos résultats confirment la nécessité de mettre en œuvre ces lignes directrices en Ontario auprès des sous-groupes de patients (sous-groupes $n^{\circ} 1$ et 4 ).

Bien que nous n'ayons pas observé de corrélation significative entre la résistance à la RMP et les antécédents de contact avec une personne atteinte de TB-MR (sous-groupe de patients $n^{\circ} 3$ ), le RC était élevé et nos chiffres étaient bas, et il semble logique que ces patients puissent présenter des risques et qu'ils fassent, de ce fait, l'objet d'analyses. Nos données soulèvent néanmoins des questions sur les avantages et les coûts potentiels liés à la réalisation d'analyses «ciblées» chez les patients du sous-groupe $n^{\circ} 2$, dans une région comme la nôtre (Toronto, Ontario) où la majorité des patients tuberculeux sont des immigrants. $\mathrm{Ce}$ critère pourrait éventuellement être modifié, en Ontario et dans les régions similaires, de manière à ce que seuls les patients originaires d'un pays à risque élevé, jeunes ou ayant récemment immigré soient soumis à des analyses. II pourrait également être envisagé de procéder à des analyses ciblées auprès des patients originaires de pays associés à un risque très élevé (région européenne).

\section{Points forts et limites}

Vu notre statut de centre de référence pour la prise en charge des cas de TB pharmacorésistante et complexe en Ontario, il n'est guère étonnant que la proportion de cas pharmacorésistants (10,9\% de TB R-INH et 11,4\% de TB-MR) que nous traitons ait été supérieure aux taux provinciaux $(8,5 \%$ et $1,4 \%)(7)$ et nationaux (6,2\% et 1,2\%) (18) en 2016 . La présence, dans notre centre, d'une population de cas pharmacorésistants supérieure à la moyenne était l'occasion d'étudier en détail les caractéristiques des patients; notre étude s'est toutefois fondée sur un nombre de cas pharmacorésistants relativement faible. De plus, il se peut que nous n'ayons pas été en mesure de déceler de corrélation significative entre certains facteurs de risque réels et la TB pharmacorésistante. II pourrait en outre y avoir un biais de sélection dans la population de notre étude, car les cas de TB-PS associés à une maladie moins grave ou à des comorbidités limitées étaient peut-être moins susceptibles d'être orientés vers notre centre spécialisé. Une autre limite possible de notre étude est qu'elle est représentative des patients de la région de Toronto (qui regroupe la majorité des cas de TB de la province; $76 \%$ en 2016) (19), et peut ne pas refléter les caractéristiques des patients des autres villes de l'Ontario, sans doute moins susceptibles d'être orientés vers notre établissement. Par ailleurs, nous ne disposions pas de renseignements précis sur tous les pays où une personne a résidé avant de venir au Canada. Enfin, nous avons analysé de nombreuses caractéristiques chez les patients pour établir leurs liens avec la TB pharmacorésistante, et il se peut que les corrélations que nous avons observées soient fausses en raison de comparaisons multiples.

\section{Conclusion}

Nous avons résumé les facteurs de risque associés à la TB $\mathrm{R}-\mathrm{INH}$ et à la TB-MR chez les patients pris en charge dans notre établissement de Toronto (Ontario). Ces résultats pourraient être utiles aux cliniciens de la province spécialisés dans la tuberculose, car ils contribuent à éclairer l'antibiothérapie empirique initiale qu'ils prescrivent dans l'attente des résultats de l'ESM phénotypique, et à faciliter la prise d'une décision quant à la nécessité de demander ou non une ESM moléculaire rapide. Ces résultats pourraient également servir d'orientation aux décideurs politiques et au personnel de laboratoire concernant l'application ciblée de l'ESM moléculaire dans la province. 


\section{Déclaration des auteurs}

T. H. - Collecte de données, analyse statistique, rédaction de la version initiale, révision, édition

N. S., P. D., J. M. et H. S. - Collecte de données

T. M. et S. B. - Collecte de données, analyse statistique, rédaction de la version initiale, révision, édition

\section{Conflit d'intérêts}

Aucun.

\section{Financement}

Ces travaux ont été financés en partie par la Fondation médicale Kurozumi, le Laboratoire Tokyo-Hokenkai Byotai-Seiri et la Fondation Takeda pour la science. Cette étude n'a bénéficié d'aucun autre financement externe.

\section{Références}

1. Minion J, Gallant V, Wolfe J, Jamieson F, Long R. Multidrug and extensively drug-resistant tuberculosis in Canada 19972008: demographic and disease characteristics. PLoS One 2013;8(1):e53466. DOl PubMed

2. World Health Organization. Global Tuberculosis Report 2018. Geneva (CH): WHO; 2018. https://apps.who.int/ medicinedocs/en/m/abstract/Js23553en/

3. Lewinsohn DM, Leonard MK, LoBue PA, Cohn DL, Daley CL, Desmond E, Keane J, Lewinsohn DA, Loeffler AM, Mazurek GH, O'Brien RJ, Pai M, Richeldi L, Salfinger M, Shinnick TM, Sterling TR, Warshauer DM, Woods GL. Official American Thoracic Society/Infectious Diseases Society of America/ Centers for Disease Control and Prevention Clinical Practice Guidelines: Diagnosis of Tuberculosis in Adults and Children. Clin Infect Dis 2017 Jan;64(2):111-5. DOI PubMed

4. Moniruzzaman A, Elwood RK, Schulzer M, FitzGerald JM. A population-based study of risk factors for drug-resistant TB in British Columbia. Int J Tuberc Lung Dis 2006 Jun;10(6):631-8. PubMed

5. Long $\mathrm{R}$, Langlois-Klassen $\mathrm{D}$. Increase in multidrug-resistant tuberculosis (MDR-TB) in Alberta among foreign-born persons: implications for tuberculosis management. Can J Public Health 2013 Jan;104(1):e22-7. PubMed

6. Statistique Canada Immigration et diversité ethnoculturelle - Faits saillants en tableaux, Recensement de 2016. Ottawa (ON) : Stats Can. https://www12.statcan.gc.ca/censusrecensement/2016/dp-pd/hlt-fst/imm/index-eng.cfm

7. Vachon J, Gallant V, Siu W. La tuberculose au Canada, 2016. Relevé des maladies transmissibles au Canada 2018;44(3/4):85-91. DOI
8. LaFreniere $\mathrm{M}$, Hussain $\mathrm{H}$, Vachon J. La résistance aux antituberculeux au Canada : 2017. Relevé des maladies transmissibles au Canada 2018;44(11):330-6. DOI

9. Brode SK, Varadi R, McNamee J, Malek N, Stewart S, Jamieson FB, Avendano M. Multidrug-resistant tuberculosis: treatment and outcomes of 93 patients. Can Respir J 2015 Mar-Apr;22(2):97-102. DOI PubMed

10. Woods GL. Susceptibility testing for mycobacteria. Clin Infect Dis 2000 Nov;31(5):1209-15. PubMed

11. Clinical and Laboratory Standards Institute. M24-A2 Susceptibility Testing of Mycobacteria, Nocardiae, and Other Aerobic Actinomycetes; Approved Standard-Second Edition. Wayne (PA): CLSI; 2011. https://clsi.org/media/1463/ m24a2_sample.pdf

12. Public Health Ontario. Infectious Disease Trends in Ontario. Toronto (ON): Ontario Agency for Health Protection and Promotion; 2020. https://www. publichealthontario.ca/dataand-analysis/infectious-disease/reportable-disease-trendsannually\#/2

13. Cattamanchi A, Dantes RB, Metcalfe JZ, Jarlsberg LG Grinsdale J, Kawamura LM, Osmond D, Hopewell PC, Nahid $P$. Clinical characteristics and treatment outcomes of patients with isoniazid-monoresistant tuberculosis. Clin Infect Dis 2009 Jan;48(2):179-85. DOI PubMed

14. Tegegne BS, Mengesha MM, Teferra AA, Awoke MA, Habtewold TD. Association between diabetes mellitus and multi-drug-resistant tuberculosis: evidence from a systematic DOI PubMed

15. Suchindran S, Brouwer ES, Van Rie A. Is HIV infection a risk factor for multi-drug resistant tuberculosis? A systematic review. PLoS One 2009;4(5):e5561. DOI PubMed

16. United Nations. Department of Economic nad Social Affairs. 2017 International Migration Report [Highlights]. New York (NY): United Nations; 2017. https://www.un.org/en/ development/desa/population/migration/publications/ migrationreport/docs/MigrationReport2017_Highlights.pdf

17. Immigration, Réfugiés et Citoyenneté Canada. Rapport annuel au Parlement sur l'immigration, 2015. https:// www.canada.ca/fr/immigration-refugies-citoyennete/ organisation/publications-guides/rapport-annue I-parlement-immigration-2015.html

18. Agence de la santé publique du Canada. Normes Canadiennes pour la lutte antituberculeuse $7^{\mathrm{e}}$ edition : 2014. Ottawa (ON) : ASPC. https://www.canada. $\mathrm{ca} / \mathrm{fr} /$ sante-publique/services/maladies-infectieuses/ normes-canadiennes-lutte-antituberculeuse-7e-edition.html

19. Public Health Ontario. Infectious Disease Trends in Ontario. Archive of 2016 summaries. Toronto (ON) : Ontario Agency for Health Protection and Promotion; November 2018. https://www.publichealthontario.ca/en/dataandanalytics/ pages/rdto.aspx\#/34 